\title{
Th2 cell hyporesponsiveness during chronic murine schistosomiasis is cell intrinsic and linked to GRAIL expression
}

\author{
Justin J. Taylor, ${ }^{1}$ Connie M. Krawczyk, ${ }^{1}$ Markus Mohrs, ${ }^{2}$ and Edward J. Pearce ${ }^{1}$ \\ 1Department of Pathobiology, School of Veterinary Medicine, University of Pennsylvania, Philadelphia, Pennsylvania, USA. \\ ${ }^{2}$ Trudeau Institute, Saranac Lake, New York, USA.
}

\begin{abstract}
Chronic infections are associated with progressively declining $T$ cell function. Infections with helminth parasites, such as Schistosoma mansoni, are often chronic and characterized by the development of strong Th2 responses that peak during the acute stage of infection and then decline despite ongoing infection; this minimizes Th2-dependent immunopathology during the chronic stage of infection. We sought to understand the basis for the decline in Th2 responses in chronic schistosomiasis. Using IL-4 reporter mice (mice that express EGFP as a reporter for $\mathrm{Il} 4$ gene expression) to identify $\mathrm{Th} 2$ cells, we found that Th2 cell numbers plateaued during acute infection and remained constant thereafter. However, the percentages of Th2 cells proliferating during late infection were strikingly lower than those during acute infection. Th2 cell hyporesponsiveness was evident within $10 \mathrm{~d}$ of initiation of the Th2 response and became progressively ingrained thereafter, in response to repeated $\mathrm{Ag}$ stimulation. Gene expression analyses implicated the E3-ubiquitin ligase gene related to anergy in lymphocytes (GRAIL) in the hyporesponsive state. Consistent with this, suppression of GRAIL expression using retrovirally delivered siRNA prevented the development of hyporesponsiveness induced by repeated Ag stimulation in vitro or in vivo. Together, these data indicate that the decline in Th2 cell responsiveness during chronic schistosomiasis is the net result of the upregulation of GRAIL expression in response to repeated Ag stimulation.
\end{abstract}

\section{Introduction}

Repeated antigenic stimulation of $\mathrm{T}$ cells is recognized to result in the development of a hyporesponsive state $(1,2)$. We are interested in the relevance of this situation to the immunological hyporesponsiveness that can develop during chronic infection, in which ongoing exposure to Ag is presumably occurring. Recent work has shown that during chronic viral infections, the presence of the regulatory cytokine IL-10 and the expression of programmed death-1 (PD-1) by $\mathrm{CD}^{+} \mathrm{T}$ cells play central roles in diminished responsiveness (2). However, the mechanisms controlling CD4+ $\mathrm{T}$ cell responsiveness in chronic infections with extracellular pathogens remain unclear. Foremost among extracellular pathogens causing chronic infections are the helminth parasites. Collectively, these organisms infect billions of people worldwide and remain a neglected and underestimated cause of serious morbidity $(3,4)$.

Schistosomiasis, an important helminth infection, afflicts 200 million people and causes more than 300,000 deaths in Africa alone each year $(5,6)$. The infection is chronic because of the ability of schistosomes to evade the immune response and survive in the host for years. Adult Schistosoma mansoni live within the portal vasculature, where female worms produce eggs that pass from the lumen of the blood vessel to the intestine, and from there to the outside, where they can continue the life cycle. However, many eggs are carried by the blood flow into the liver, where they become trapped in the sinusoids and induce a highly polarized Th2 response $(7,8)$. The Th2 response, while unable to eradicate

Conflict of interest: The authors have declared that no conflict of interest exists. Nonstandard abbreviations used: GRAIL, gene related to anergy in lymphocytes; hp, hairpin; PD-1, programmed death-1; SEA, schistosome egg Ag.

Citation for this article: J. Clin. Invest. 119:1019-1028 (2009). doi:10.1172/JCI36534. the worms, orchestrates granuloma formation around tissuetrapped eggs and alternatively activates macrophages, both functions essential for host survival (9-14). However, the Th2 cytokine IL-13 plays a major role in the development of hepatic fibrosis (12, $15,16)$, which appears to be an inevitable consequence of having to make a Th2 response in order to survive infection.

It has been long recognized that during schistosomiasis, the parasite-induced Th response, as measured by in vitro Ag-stimulated $\mathrm{T}$ cell proliferation or cytokine secretion assays, peaks early and then declines despite ongoing infection (7, 17-19). This process is referred to as immunomodulation and is also apparent in other chronic helminth infections (20). The size of granulomatous lesions around parasite eggs trapped in host tissues mirrors the rise and fall of the Th response (17-19). In contrast to other chronic infections, the reduction in immune responsiveness during schistosomiasis does not contribute to pathogen survival, but instead acts to minimize immunopathology in a setting where the immune system is incapable of eliminating the pathogen $(17,21,22)$.

Despite prolonged interest in immunomodulation during schistosomiasis, the cellular and/or molecular basis for this process has yet to be defined. An important unresolved issue is the fate of the Th2 cell population as the infection progresses into the chronic phase. Several groups have reported increased Th cell apoptosis during infection (23-25), leading some to hypothesize that Th2 cell death accounts for the diminished proliferative and cytokine responses in chronic infection. Other groups have suggested that Th2 cells are present but rendered inactive either through illdefined cell-intrinsic mechanisms $(26,27)$ or as a result of extrinsic factors, such as the presence of IL-10 (28-30) or altered Ag presentation (31). These studies were limited by their inability to directly quantitate and characterize Th2 cells induced by the infection. 
A

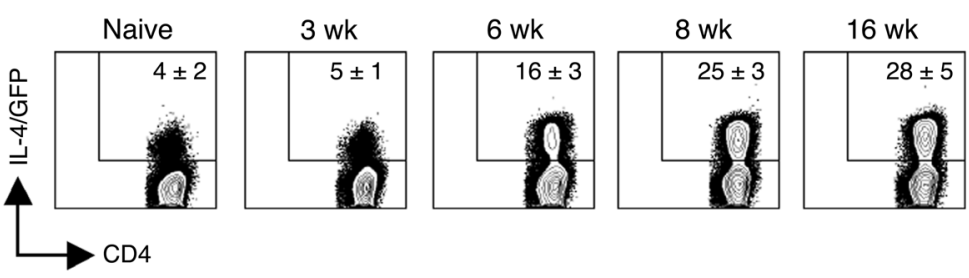

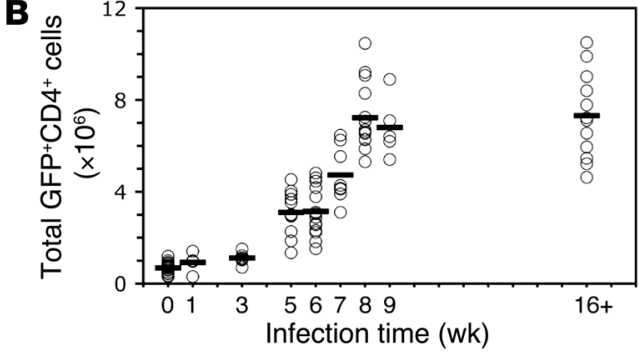

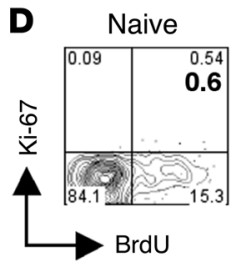

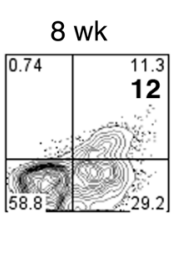
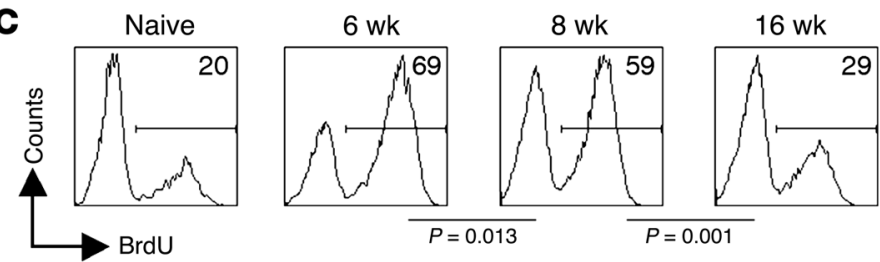

\section{Figure 1}

The Th2 population is maintained but hyporesponsive during chronic schistosome infection. (A) At 3, 6, 8, and 16 wk after infection, CD4+ splenocytes from control uninfected 4 get mice, as well as mice infected with $S$. mansoni for the indicate times, were analyzed ex vivo for the expression of IL-4/GFP by flow cytometry. Numbers within plots indicate mean \pm SD percent CD4+ cells that are IL-4/GFP+ (3-5 mice per group). (B) Total number of Th2 cells in the spleen, determined using the percentages measured by flow cytometry coupled with spleen cell numbers ascertained by counting. Data points are from individual mice from 6 independent experiments; horizontal lines indicate mean values. (C) BrdU incorporation into gated GFP+CD4+ cells in the spleens of naive control mice, as well as mice infected with $S$. mansoni for the indicated times, was assessed ex vivo following a 7-d labeling period in vivo. Numbers within histograms indicate mean percent BrdU+ cells (3-4 mice per group). (D) Same as in C, except Ki-67 expression was analyzed in addition to BrdU incorporation after a 5 -d BrdU labeling period in vivo. Numbers within plots indicate percent cells in the respective quadrants. Bold numbers indicate mean percent Ki- $67^{+}$cells (3-4 mice per group). Plots and quadrant statistics are from representative animals.

Here we have reexamined the hyporesponsiveness to egg Ag that develops during chronic schistosomiasis. Using the 4get mouse, which expresses EGFP as a reporter for Il4 gene expression (32, 33 ), we found that the reduced immunological responsiveness during chronic schistosomiasis was not the result of a loss of Th2 cells, but rather caused by the development of cell-intrinsic hyporesponsiveness in a persisting population. Our data indicate that the expression of gene related to anergy in lymphocytes (GRAIL), an E3 ubiquitin ligase previously implicated in the development of $\mathrm{T}$ cell anergy $(34,35)$, is important in the hyporesponsiveness that develops over time with repeated antigenic stimulation. To our knowledge, this is the first demonstration of a role for GRAIL in a system in which $\mathrm{CD} 4^{+} \mathrm{T}$ cell hyporesponsiveness is a natural consequence of chronic infection.

\section{Results}

The Th2 population rapidly expands to reach a plateau and then fails to contract. Previous reports have shown that during schistosome infection, the potent schistosome egg Ag-specific (SEA-specific) Th2 response, which develops rapidly after exposure to parasite eggs, declines over time despite ongoing infection $(7,17-19)$. We examined ex vivo SEA-driven proliferation and cytokine production and observed the same pattern of rising and then declining responsiveness (data not shown and Supplemental Figure 1; supplemental material available online with this article; doi:10.1172/ JCI36534DS1). To examine whether loss of responsiveness reflects the contraction of the Th2 population over time, we infected 4get mice and at various times after infection used flow cytometry to quantitate $\mathrm{GFP}^{+} \mathrm{CD} 4^{+} \mathrm{Th} 2$ cells within responding lymphoid organs. Th2 cell numbers were not significantly different from background at $1 \mathrm{wk}$ and $3 \mathrm{wk}$, prior to parasite maturation, but increased at $5 \mathrm{wk}$, soon after the onset of egg production, and continued to increase until $8 \mathrm{wk}$, at which time about $25 \%$ of splenic $\mathrm{CD} 4^{+} \mathrm{T}$ cells were Th2 (Figure 1, A and B). These kinetics for the development of the Th2 response are in agreement with published studies in which splenocytes were restimulated in vitro with SEA and secreted Th2 cytokines were measured (7). Despite observed decreases in Th2 cell responsiveness at later times during infection (Supplemental Figure 1), we found that the percentage and total number of Th 2 cells in the spleen were similar at $8 \mathrm{wk}$ (Figure $1, \mathrm{~A}$ and $\mathrm{B}$ ) and at all time points examined thereafter, up to 37 wk (data not shown). The same result was observed in cells of the mesenteric $\mathrm{LN}$, another lymphoid organ intimately involved in the immune response (data not shown).

The proliferative response of Th2 cells is modulated during infection. The finding that the number of Th2 cells plateaued and remained constant thereafter (Figure 1B) suggests that observed differences in $\mathrm{CD}^{+} \mathrm{T}$ cell proliferation (Supplemental Figure 1) reflect the development of nonresponsiveness in persisting Th2 cells. To explore this possibility in vivo, naive and infected animals were treated with BrdU for $7 \mathrm{~d}$, and BrdU incorporation into Th2 cells was measured by flow cytometry. We found that approximately $69 \%$ of Th2 cells in infected mice proliferated between 5 and $6 \mathrm{wk}$ of infection, about $59 \%$ proliferated between 7 and $8 \mathrm{wk}$, and about $29 \%$ proliferated between 15 and $16 \mathrm{wk}$ (Figure 1C). The level of proliferation at $15-16$ wk was close to background values, since approximately $20 \%$ of the small number of Th2 cells in naive mice incorporated BrdU over a 7-d labeling period (Figure 1C).

As another measure of proliferation, we looked for the expression of nuclear cell proliferation $\mathrm{Ag} \mathrm{Ki}-67$, which is expressed in 

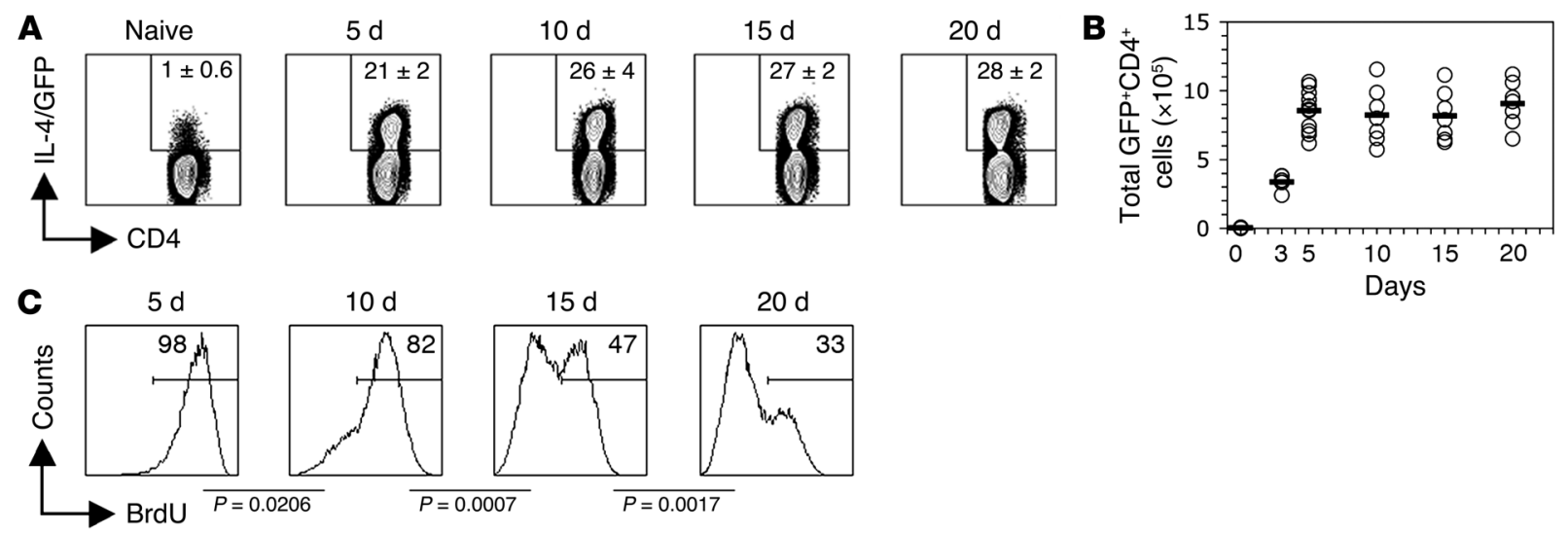

Figure 2

Chronic stimulation with SEA induces Th2 hyporesponsiveness. (A) Mice infected with S. mansoni received 1 or more egg injections. Repeat egg injections were $5 \mathrm{~d}$ apart, such that mice for the 20-d time point had received 4 injections prior to sacrifice. At $5,10,15$, and $20 \mathrm{~d}$ after the first immunization, CD4 ${ }^{+} \mathrm{T}$ cells from draining popliteal LNs were analyzed ex vivo for the expression of IL-4/GFP by flow cytometry. Numbers within plots indicate mean \pm SD percent of $\mathrm{CD} 4^{+}$cells that were IL-4/GFP+ (3 mice per group). (B) Total number of Th2 cells, determined using the percentages measured by flow cytometry coupled with spleen cell numbers ascertained by counting. Data points are from individual mice from 4 independent experiments; horizontal lines indicate mean values. (C) BrdU incorporation into draining LN GFP ${ }^{+}$CD4 ${ }^{+}$cells was assessed ex vivo after a 5 -d labeling period in vivo. Numbers within histograms indicate mean percent cells that incorporated BrdU ( 3 mice per group).

cells that are actively engaged in cell cycle (36). We found that the proportion of Th2 cells that were $\mathrm{Ki}-67^{+}$was substantially greater at $8 \mathrm{wk}$ than at $16 \mathrm{wk}$ of infection (about $12 \%$ versus about $3 \%$; Figure 1D). Barely detectable levels of Ki-67 were evident in Th2 cells from naive mice (Figure 1D). As expected, Ki-67 was exclusively expressed by a subpopulation of Th2 cells that had incorporated BrdU (Figure 1D).

We also analyzed the Th2 response in the major tissue site of egg deposition, the liver. Over $50 \%$ of $\mathrm{CD}^{+} \mathrm{T}$ cells isolated from the liver at $8 \mathrm{wk}$ of infection were Th2 cells, and this percentage did not change significantly by 16 wk of infection (Supplemental Figure 2A). Consistent with findings from the spleen (Figure 1), the proliferative response of Th2 cells in the liver was higher at the earlier time point (Supplemental Figure 2B).

Repeated exposure to SEA rapidly induces Th2 byporesponsiveness. We noticed that BrdU incorporation into Th2 cells was significantly higher 5-6 wk than 7-8 wk after infection (Figure 1C), which suggests that hyporesponsiveness develops early after onset of egg production, the stimulus for the Th2 response, and becomes more entrenched in the responding Th 2 cell population as the infection progresses. Egg production begins sometime between 4 wk and $5 \mathrm{wk}$ of infection, but the exact timing of this event is difficult to determine, because the sexual maturation of schistosomes is asynchronous and variable. To circumvent this problem and to explore in more detail the kinetics of the development of hyporesponsiveness, we adopted a model in which Th2 responses are induced in mice by the injection of purified schistosome eggs $(37,38)$. This system provides the advantage of a known start point for the developing immune response. In an effort to more accurately mimic the continuous deposition of eggs during infection, we gave repeated egg injections every $5 \mathrm{~d}$ for the duration of the experiment. In this system, the Th2 response, measured as the percentage or total number of $\mathrm{CD}^{+} \mathrm{T}$ cells that expressed GFP in the draining $\mathrm{LN}$, peaked by $5 \mathrm{~d}$ and was maintained thereafter (Figure 2, A and B). In contrast, after 1 injection of eggs, the Th2 population peaked at $5 \mathrm{~d}$ and subsequently contracted (data not shown), which indicates that the continued deposition of eggs is essential to maintain the Th2 population. To test the proliferative response of Th2 cells in egg-injected mice, we labeled the cells in vivo with BrdU for a 5-d period prior to sacrifice at different time points. We found that early in the course of the egg-induced response, essentially all Th2 cells proliferated (Figure 2C), but that with maintained exposure to eggs, the percentage of proliferating Th2 cells declined; by $20 \mathrm{~d}$, only $33 \%$ of the cells induced by the response incorporated BrdU (Figure 2C). These data indicate that Th2 hyporesponsiveness begins to develop within $10 \mathrm{~d}$ of the initiation of the Th2 response and becomes progressively ingrained thereafter.

Th2 cells from infected mice are intrinsically byporesponsive. We investigated whether hyporesponsiveness is Th2 cell intrinsic or imposed by extrinsic suppressive mechanisms. To distinguish between these possibilities, we sorted Th2 cells from $7 \mathrm{wk}$ or 15 wk infected Thy1.1 mice and transferred them into naive, $7 \mathrm{wk}$ infected, or 15 wk infected Thy 1.2 congenic recipients, allowing us to directly compare the proliferative response of donor and recipient Th2 cells within the same animal by measuring incorporation of BrdU into the Thy $1.1^{+}$(donor) and Thy1.1- (recipient) Th2 cells. We found that Th 2 cells from $7 \mathrm{wk}$ or $15 \mathrm{wk}$ infected mice failed to proliferate when transferred into uninfected mice (Figure 3, A and D), confirming that the proliferation of Th2 cells in infected animals is Ag dependent. The proliferative response of Th 2 cells from $15 \mathrm{wk}$ infected donor mice transferred into $7 \mathrm{wk}$ infected recipient mice was no greater than that of Th2 cells that remained within the 15 wk setting (Figure 3, B and C). As a control, Th2 cells from $15 \mathrm{wk}$ infected donors were transferred into $15 \mathrm{wk}$ infected recipients, where they remained hypoproliferative (Figure 3C). Thus, Th2 cells from $15 \mathrm{wk}$ infected mice did not regain responsiveness by transfer into $7 \mathrm{wk}$ infected recipient mice, an environment known to be capable of supporting Th 2 cell proliferation. In the converse experiment, we transferred the more functional Th2 cells found in $7 \mathrm{wk}$ infected mice into $15 \mathrm{wk}$ infected animals and found that over the 7-d BrdU labeling period, these cells continued to proliferate as well as did those transferred into $7 \mathrm{wk}$ infected 


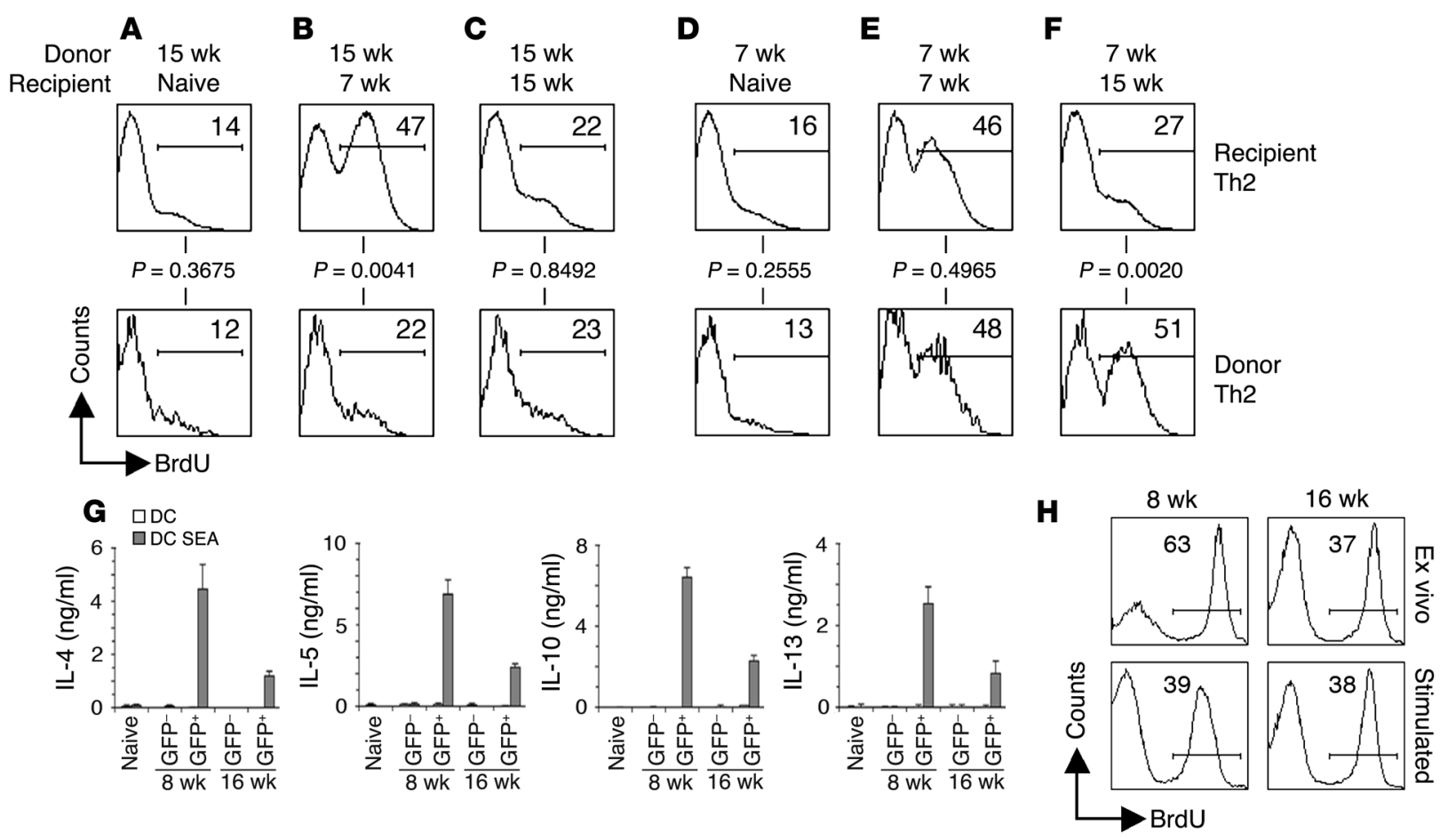

\section{Figure 3}

Cell-intrinsic Th2 hyporesponsiveness is induced by repeated stimulation with antigen. (A-F) GFP+CD4+ cells, sorted from $4 \mathrm{get}$ Thy 1.1 mice infected with S. mansoni for 15 wk (A-C) or 7 wk (D-F), were adoptively transferred into naive (A and D), 7 wk infected (B and E), or 15 wk infected (C and F) congenic Thy 1.1- recipients $1 \mathrm{~d}$ prior to BrdU labeling for $7 \mathrm{~d}$. Proliferation of donor and recipient Th2 cells was assessed using flow cytometry to measure the incorporation of BrdU. Panels represent concatenated data from all mice within the experiment (3 mice per group). Numbers within histograms indicate mean percent BrdU+ cells. (G) Sorted GFP+CD4+ and GFP-CD4+ cells from naive, 8 wk infected, or 16 wk infected animals were restimulated in vitro with DCs pulsed with or without SEA for $72 \mathrm{~h}$. Cytokine concentrations in culture supernatants were measured by ELISA. Error bars denote SD of 3 measurements per group. (H) Sorted GFP+CD4+ cells from 8 wk or 16 wk infected mice were restimulated in vitro with SEA-pulsed DCs for $72 \mathrm{~h}$ directly ex vivo, or for $72 \mathrm{~h}$ after an 8-d in vitro period during which they were continuously exposed to SEA-pulsed DCs. Numbers within histograms indicate percent BrdU+ cells.

recipients (Figure 3, E and $\mathrm{F}$ ), which indicates that the environment in mice infected for $15 \mathrm{wk}$ is capable of supporting the proliferation of responsive Th2 cells.

These data indicate that robust Ag presentation is ongoing within the $16 \mathrm{wk}$ infection setting, suggesting that hyporesponsive Th 2 cells are receiving activation signals but are subsequently failing to proliferate. To examine this issue, we measured the surface expression of CD62 ligand (CD62L) and CD69, markers known to be downregulated and upregulated, respectively, on activated T cells $(39,40)$. We found that the majority of $\mathrm{GFP}^{+}$ Th2 cells at both $8 \mathrm{wk}$ and $16 \mathrm{wk}$ - which effectively modeled acute and chronic infection, respectively - expressed low levels of CD62L and high levels of CD69, with little difference in terms of the expression of these markers between Th2 cells at these 2 time points (Supplemental Figure 3). These data are consistent with Th2 cells in chronically infected mice having recently received activation signals and indicate that the relatively poor proliferative response of these cells compared with that of cells from acutely infected mice is not mechanistically linked to a failure of Ag presentation. This conclusion is supported by findings from in vitro experiments, in which isolated Th2 cells from $8 \mathrm{wk}$ or $16 \mathrm{wk}$ infected mice retained their differences in responsiveness when activated by a normalized population of highly competent APCs. For these experiments, we cultured FACS-purified
$\mathrm{GFP}^{+} \mathrm{CD}^{+}$and $\mathrm{GFP}^{-} \mathrm{CD} 4^{+}$cells from infected mice for $72 \mathrm{~h}$ with SEA-pulsed bone marrow-derived DCs and measured proliferation and cytokine production. This protocol allowed for removal of potential sources of regulation, including Tregs, B cells, and macrophages, and Ag presentation was assured. Under these conditions, Th2 cells from $16 \mathrm{wk}$ infected mice made substantially lower amounts of IL-4, IL-5, IL-10, and IL-13 than did Th2 cells from $8 \mathrm{wk}$ infected mice (Figure $3 \mathrm{G}$ ) and were less proliferative, based on the incorporation of BrdU during the culture period (Supplemental Figure 4). Naive $\mathrm{CD}^{+} \mathrm{T}$ cells and $\mathrm{GFP}^{-} \mathrm{CD} 4^{+}$ $\mathrm{T}$ cells from infected mice failed to make any Th 2 cytokines (Figure $3 G$ ) or to proliferate extensively (Supplemental Figure 4) in response to SEA. When sorted, the low numbers of $\mathrm{GFP}^{+} \mathrm{CD}^{+}$ cells from naive mice showed no response to DCs with or without the addition of $\mathrm{Ag}$ (data not shown). The addition of IL-2 to these cultures did not result in an increase in proliferation or cytokine production by $\mathrm{GFP}^{+}$or $\mathrm{GFP}^{-}$cells (data not shown).

Our findings from in vivo experiments suggested that the development of Th2 hyporesponsiveness would be inducible by repeated stimulation of Th 2 cells with $\mathrm{Ag}$ in vitro. To test this, we purified Th 2 cells from $8 \mathrm{wk}$ and $16 \mathrm{wk}$ infected animals and analyzed their ability to proliferate immediately ex vivo and after repeated stimulation with SEA-pulsed DCs. After repeated stimulation, Th2 cells were sorted and replated with SEA-pulsed DCs for 
A
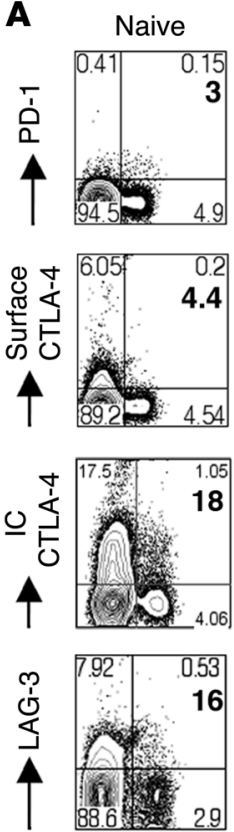

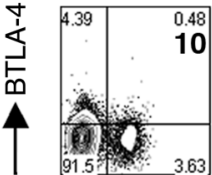

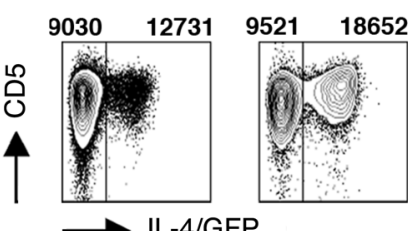

8 wk
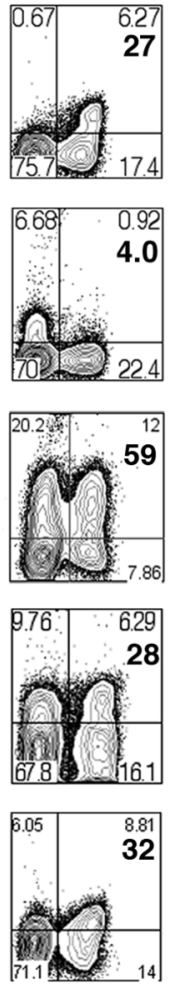

652
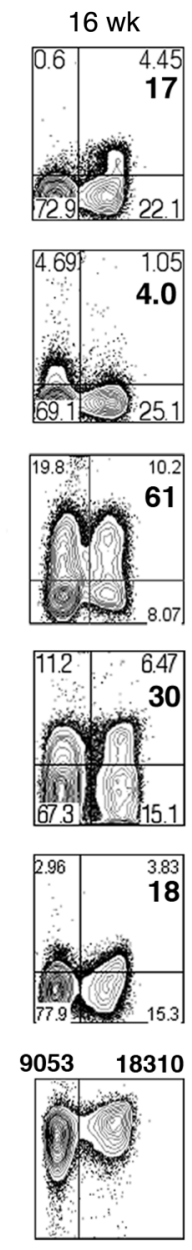

B
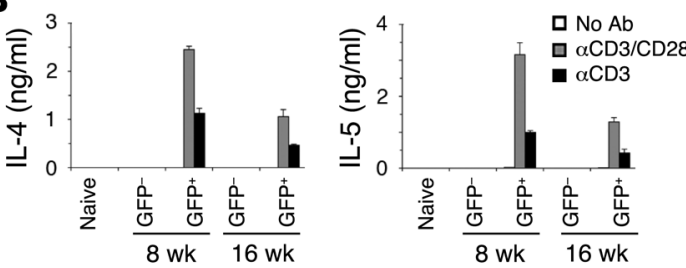

c

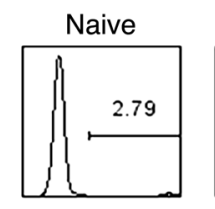

8 wk GFP+

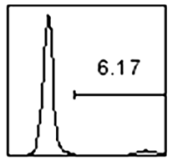

16 wk GFP+
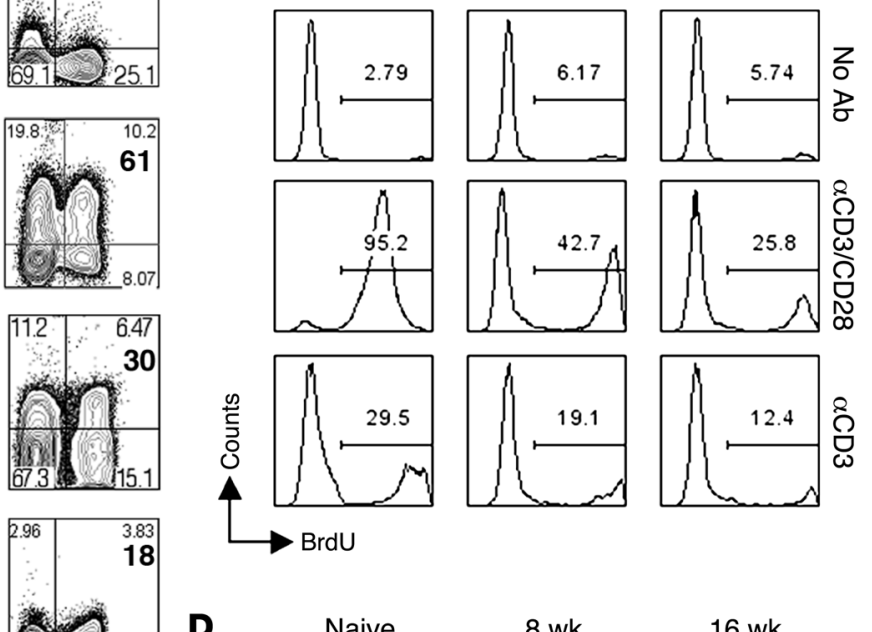

D
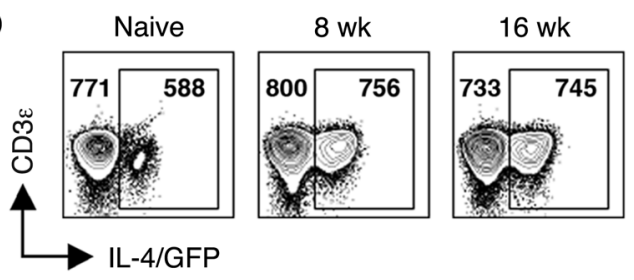

\section{Figure 4}

Th2 hyporesponsiveness does not rely on the ligation of inhibitory receptors. (A) Gated CD4+ splenocytes from infected and control mice were analyzed for the expression of PD-1, surface CTLA-4, intracellular (IC) CTLA-4, LAG-3, BTLA-4, CD5, and IL-4/GFP by flow cytometry. Plots shown and quadrant statistics are from representative animals (3-4 mice per group). For all plots but CD5, numbers within plots indicate percent cells in the respective quadrants, and bold numbers indicate mean percent IL-4/GFP+ cells expressing the marker. For CD5, numbers above plots indicate mean MFI. (B and C) Sorted GFP+CD4+ and GFP-CD4+ cells from naive mice or mice infected with $\mathrm{S}$. mansoni for 8 wk or 16 wk were restimulated in vitro with plate-bound anti-CD3 alone or with anti-CD28 for $72 \mathrm{~h}$. Control mice received no antibody. (B) Cytokine concentrations in culture supernatants were measured by ELISA. Error bars denote SD of 3 measurements per group. (C) Proliferation was assessed using flow cytometry to determine the incorporation of BrdU during the 72-h culture period. Numbers within histograms indicate percent BrdU ${ }^{+}$cells. (D) Gated CD4+ splenocytes from infected and control mice were analyzed for the expression of CD3 $\varepsilon$ and IL-4/GFP by flow cytometry. Plots are from representative animals. Numbers within plots indicate mean CD3 $\varepsilon$ MFI within IL-4/GFP+ and IL-4/GFP- cells (3-4 mice per group).

$72 \mathrm{~h}$ in the presence of BrdU. The in vitro-stimulated cells from the $8 \mathrm{wk}$ infected mice were rendered hypoproliferative by repeated stimulation compared with Th2 cells examined ex vivo (39\% versus $63 \%$ of Th2 cells incorporated BrdU; Figure $3 \mathrm{H}$ ). In contrast, Th2 cells from $16 \mathrm{wk}$ infected mice were not similarly sensitive to prolonged culture with Ag (Figure $3 \mathrm{H}$ ), consistent with these populations having already attained maximal hyporesponsiveness in vivo. This view fits our finding that the proportion of Th 2 cells that incorporate BrdU was similar at $16 \mathrm{wk}$ of infection and at later time points (data not shown). Taken together, the data from both in vivo and in vitro experiments concur in suggesting that as schistosome infection progresses, Th2 cells become intrinsically hyporesponsive in response to repeated Ag stimulation.
Th2 cell byporesponsiveness is not the result of the ongoing delivery of inhibitory signals by APCs. Based on recent findings with exhausted $\mathrm{CD}^{+} \mathrm{T}$ cells $(1,2)$, we reasoned that differential expression of inhibitory receptors could account for the diminished responsiveness of Th2 cells found at $16 \mathrm{wk}$ (Figure 3 ). To address this, we used flow cytometry to measure surface expression of a panel of inhibitory receptors that have been implicated in $\mathrm{T}$ cell nonresponsiveness and/or exhaustion. However, we found that PD-1, CTLA-4, LAG-3, BTLA-4, and CD5 were all expressed at similar or lower levels on Th2 cells from 16 wk versus 8 wk of infection (Figure 4A), which suggests that expression of these receptors per se is insufficient to confer hyporesponsiveness. To bypass the possibility that Ag-pulsed DCs ligate uncharacterized inhibitory 

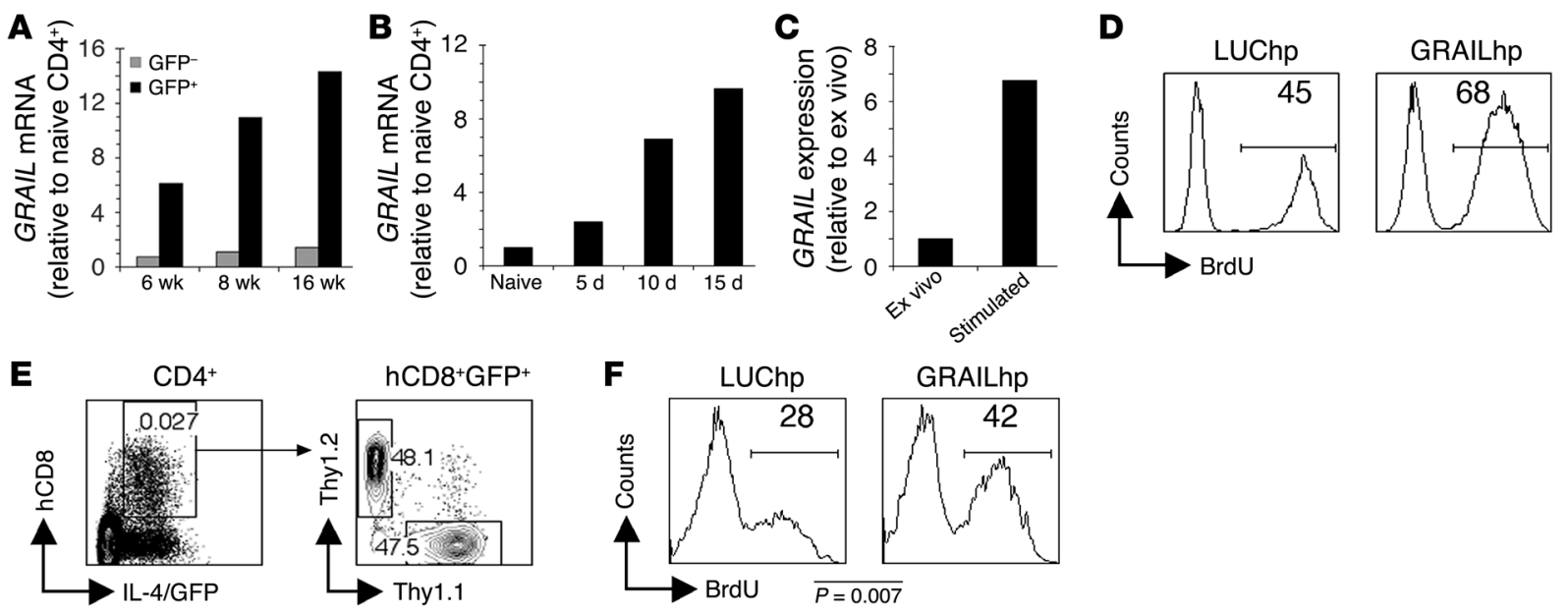

\section{Figure 5}

Increased GRAIL underlies Th2 cell hyporesponsiveness. (A) At 6, 8, and 16 wk after infection, GFP+CD4+ and GFP-CD4+ cells from S. mansoni-infected and $\mathrm{CD}^{+}$cells from uninfected 4 get mice were analyzed by real-time RT-PCR for GRAIL mRNA. (B) At 5, 10, and 15 d after initiation of egg injections (described in Figure 2), draining LN GFP+CD4+ cells from infected mice and CD4+ cells from naive control 4get mice were analyzed by real-time RT-PCR for GRAIL mRNA. (C) GFP+CD4+ cells from acutely infected mice were analyzed by real-time RT-PCR for GRAIL expression ex vivo or after $8 \mathrm{~d}$ in vitro incubation with SEA-pulsed DCs. (D) GFP+CD4+ cells from 8 wk infected mice were infected with GRAILhp or LUChp prior to $8 \mathrm{~d}$ stimulation with SEA-pulsed DCs. After stimulation, sorted hCD8+ cells were restimulated for $72 \mathrm{~h}$ with SEApulsed DCs. In vitro proliferation was assessed using flow cytometry to determine the incorporation of BrdU during the 72-h culture. Numbers within histograms indicate mean percent BrdU+ cells. (E and F) GFP+CD4+ cells from 4get Thy 1.2 or 4 get Thy 1.1 mice $5 \mathrm{~d}$ after initiation of egg injections were infected with retrovirus prior to 1-d stimulation with SEA-pulsed DCs. Cells were then mixed and transferred into infected Balb/c mice. (E) Gating strategy used to detect hp-expressing cells in recipient animals. Numbers within plots represent the frequency of the cells within the gate. (F) BrdU incorporation into Thy1.1+ (GRAILhp) and Thy1.2+ (LUChp) CD4+IL-4/GFP+hCD8+ cells was analyzed on day 11 , after a 3-d BrdU labeling period. Shown are concatenated data from all mice within the experiment (4-5 mice per group). Numbers within histograms represent mean percent $\mathrm{BrdU}+$ cells.

receptors preferentially expressed on hyporesponsive Th2 cells, we stimulated Th2 cells sorted from acutely or chronically infected mice with anti-CD3/CD28 antibodies in the absence of APCs and measured cytokine production and proliferation. After stimulation with anti-CD3 with or without anti-CD28, Th2 cells from chronically infected mice produced less IL-4 and IL-5 (Figure 4B) and incorporated less BrdU (Figure 4C) than did Th2 cells from acutely infected mice. This was not the result of reduced expression of TCR on hyporesponsive Th2 cells, because CD3 levels on Th2 cells from $8 \mathrm{wk}$ and $16 \mathrm{wk}$ infections were indistinguishable (Figure 4D). Taken together, these data support the view that Th2 cells are not being rendered hyporesponsive by ongoing ligation of inhibitory receptors.

Th2 cell hyporesponsiveness is imposed by elevated expression of GRAIL. In a microarray-based comparison of gene expression in naive $\mathrm{CD} 4^{+}$ $\mathrm{T}$ cells versus Th 2 cells from $16 \mathrm{wk}$ infected mice, the E3 ubiquitin ligase GRAIL was found to be highly upregulated in Th2 cells from the infected animals (data not shown). The fact that GRAIL upregulation has previously been shown to be important for the induction of $\mathrm{T}$ cell anergy in vitro and in vivo $(34,35)$ made it an attractive target for further study. Real-time RT-PCR revealed that GRAIL expression was apparent in Th2 cells sorted from $6 \mathrm{wk}$ of infection, the early phase of the egg-induced response, and expression increased as the infection progressed into late chronicity (Figure 5A). GRAIL expression did not increase over time in GFP-CD4 ${ }^{+}$ $\mathrm{T}$ cells from the same infected animals (Figure 5A). Because a percentage of Th2 cells from $6 \mathrm{wk}$ infected mice were already hyporesponsive (Figure $1 \mathrm{C}$ ), these data may suggest a role for GRAIL in the development of hyporesponsiveness. However, another possibility is that GRAIL is highly expressed in Th2 cells in general. To address this issue, we examined GRAIL expression in Th2 cells sorted from egg-injected animals. We found that Th2 cells isolated from the draining LN $5 \mathrm{~d}$ after egg injection expressed twice the level of GRAIL mRNA compared with their naive $\mathrm{CD}^{+}$counterparts (Figure 5B). Analysis of Th 2 cells at $10 \mathrm{~d}$ and $15 \mathrm{~d}$ of repeated egg injections revealed that GRAIL expression in Th2 cells progressively and markedly increased over time (Figure 5B). Additionally, repeated stimulation of Th2 cells from $8 \mathrm{wk}$ infected mice in vitro for $8 \mathrm{~d}$, a process we found to induce hyporesponsiveness (Figure 3I), led to an increase in GRAIL expression (Figure 5C). Taken together, the results indicate that GRAIL upregulation in Th2 cells correlates with the progressive development of hyporesponsiveness that occurs as a result of chronic stimulation with Ag.

To test whether increased GRAIL expression is responsible for diminished proliferative potential in Th2 cells, we used a retrovirus encoding a shRNA specific for GRAIL (referred to herein as hairpin GRAIL [GRAILhp]) to suppress GRAIL expression. In this system, successfully transduced cells express human CD8 (hCD8), and in these cells GRAIL expression was suppressed by approximately $80 \%$ compared with cells expressing a control luciferase shRNA (LUChp; Supplemental Figure 5). To test whether GRAIL knockdown blocked the development of Th2 hyporesponsiveness, we FACS purified Th 2 cells from $8 \mathrm{wk}$ infected mice and transduced them with GRAILhp or LUChp retrovirus immediately prior to repeated stimulations with SEA-pulsed DCs for $8 \mathrm{~d}$. After stimulation, $\mathrm{GFP}^{+} \mathrm{hCD}^{+}$cells were purified and replated with SEA- 
pulsed DCs in the presence of BrdU for $72 \mathrm{~h}$. As expected, Th2 cells expressing the control LUChp became hyporesponsive as a result of prolonged culture with SEA-pulsed DCs (Figure 5D). Strikingly, GRAIL knockdown effectively prevented the development of the hyporesponsiveness in this context (Figure 5D). Indeed, Th2 cells expressing the GRAILhp incorporated levels of BrdU similar to those of Th2 cells collected from 8 wk infected mice and stimulated immediately ex vivo (compare Figure 3 and Figure 5D). These data indicate that the hypoproliferative state induced by prolonged stimulation with Ag is dependent on the upregulation of GRAIL and suggest that GRAIL expression underlies Th2 hyporesponsiveness induced by repeated stimulation with Ag during chronic infection. Interestingly, while GRAIL knockdown consistently prevented the development of a hypoproliferative state, it did not allow increased cytokine production by repeatedly stimulated Th2 cells (data not shown).

We additionally analyzed whether GRAIL could limit Th2 cell proliferation in infected animals. To do this we FACS purified Th2 cells from 4get Thy1.1 and 4get Thy 1.2 mice $5 \mathrm{~d}$ after egg injection (at which time most Th2 cells were proliferative; Figure 2C) and transduced them with GRAILhp and control LUChp retrovirus, respectively. Transduced 4get Thy1.1 and 4get Thy1.2 Th2 cells were mixed and adoptively transferred into Balb/c mice that had been infected more than $10 \mathrm{wk}$ earlier. Using this protocol, we were able to compare LUChp- and GRAILhp-expressing Th2 cells within the same infected hosts. At $8 \mathrm{~d}$ following transfer, mice were treated with BrdU for $3 \mathrm{~d}$, after which splenocytes were recovered, and Thy $1.1^{+}$and Thy $1.2^{+}$cells within the $\mathrm{hCD} 8^{+} \mathrm{GFP}^{+} \mathrm{CD} 4^{+}$population (Figure 5E) were analyzed for the presence of BrdU. Although $28 \%$ of LUChp-transduced Th2 cells incorporated BrdU, 42\% of GRAILhp-transduced Th2 cells incorporated BrdU, a highly statistically significant difference in proliferation $(P=0.007$; Figure $5 \mathrm{~F})$. These in vivo findings support the view that GRAIL is an important regulator of Th2 hyporesponsiveness during infection. Together, these data indicate that prolonged stimulation with $\mathrm{Ag}$ as a result of chronic infection leads to Th2 cell hyporesponsiveness and point to GRAIL as an important mediator of this process.

\section{Discussion}

Here we have explored the underlying basis of the diminished Th2 responses that characterize infection with an extracellular pathogen, S. mansoni. Using IL-4 reporter 4get mice, we found that the number of Th2 cells increased dramatically after exposure to parasite eggs, the Ag stimulus for Th2 response development, and then reached a plateau that remained relatively stable thereafter. Despite ongoing exposure to Ag, Th2 cells became progressively less proliferative over time, such that the proliferative capacity of Th2 cells in chronically infected animals was significantly reduced compared with earlier times during infection (Figure 1C). This functional deficit developed in response to continued Ag stimulation, was Th2 cell intrinsic, and correlated with increased expression of the E3 ubiquitin ligase GRAIL. A central role for GRAIL in the hyporesponsiveness that characterizes immunomodulation during chronic schistosomiasis is likely, since RNAi-mediated suppression of GRAIL diminished the development of Th2 cell hyporesponsiveness after prolonged Ag stimulation.

The consensus in the schistosomiasis field is that $\mathrm{T}$ cell responses are maximal in infected mice $8 \mathrm{wk}$ after infection (41), a time point 3-4 wk after the onset of parasite egg production. Our data support this view in part: the number of Th2 cells within responding lymphoid organs peaked at this time point. However, our data also showed clearly that about half of these Th2 cells did not incorporate BrdU and that higher percentages of Th2 cells were proliferating 2 wk prior to this time point, which indicates that hyporesponsive Th2 cells begin to accumulate early in the development of the Th2 response. Strong support for this view comes from the results of our egg injection studies, which showed that the proliferative response of Th2 cells diminished as the response progressed in time, and from the observation that the proliferative ability of Th2 cells from acutely infected mice diminished after stimulation with Ag-pulsed DCs in vitro for an extended period. These results suggest that hyporesponsiveness within the Th2 population is a dynamic process that begins early in the response and becomes more pervasive the longer cells are stimulated.

Recent reports have identified the persistence but functional impairment (i.e., exhaustion) of Ag-specific $\mathrm{CD}^{+} \mathrm{T}$ cells in other chronic infection settings (2). $\mathrm{CD}^{+} \mathrm{T}$ cell exhaustion in these mice appears to rely on the presence of IL-10 and the expression of PD-1, as blockade of either in infected animals restored T cell function, and elimination of the virus ensued (reviewed in ref. 2). In our present study, the expression of PD-1 and the production of IL-10 by Th2 cells was reduced later in infection, which argues against a role for either of these molecules in Th2 hyporesponsiveness. Consistent with this view, blockade of PD-1 or the IL-10 receptor did not result in the restoration of Th2 cytokine production or proliferation (data not shown). Our data support the conclusion that the mechanisms underlying $\mathrm{CD}^{+} \mathrm{T}$ cell exhaustion during chronic viral infection and Th2 cell hyporesponsiveness in chronic helminth infection are distinct. Consistent with this, GRAIL expression is not upregulated in exhausted CD8 ${ }^{+} \mathrm{T}$ cells (42).

Several reports have developed the view that immunomodulation during schistosomiasis is basically the result of T cell anergy (e.g., refs. 26, 27). Classically, anergy is induced in vitro through the activation of Th cells through the TCR in the absence of costimulation. However, a form of $\mathrm{T}$ cell hyporesponsiveness also develops in mice after prolonged exposure to $\mathrm{Ag}$, and while this condition is often referred to as anergy as well, there has been a move to reclassify it as adaptive tolerance (1). This condition differs from classic anergy in its requirement for Ag persistence in order to maintain the nonresponsive state and in that responsiveness is not recovered after addition of IL-2. We perceive chronic schistosomiasis as a pro-adaptive tolerance setting, because Ag is in constant supply as the parasites persist and continue to produce eggs, and there is the opportunity for ongoing $\mathrm{T}$ cell activation. Consistent with this view, drug treatment of chronically infected patients, and therefore the removal of $\mathrm{Ag}$, results in a return of $\mathrm{T}$ cell responsiveness, with PBMCs from cured patients remaining highly responsive to schistosome Ag for decades following chemotherapy (21).

GRAIL has previously been shown to be critical for the development of anergy induced by the activation of T cells in the absence of costimulation in vitro (34) and in vivo after immunization with soluble peptide or superantigen (35). To our knowledge, our report is the first to show that GRAIL is expressed in hyporesponsive Th2 cells that have developed as a result of chronic infection. In other systems, retrovirally imposed GRAIL overexpression has been shown to potently suppress proliferation $(35,43)$, a finding consistent with our observations. How GRAIL functions to prevent proliferation is not fully understood. However, a recent report showed that Rho GDP-dissociation inhibitor (RhoGDI) family members are ubiquitinated by GRAIL (44). RhoGDIs are regulators of Rho, 
which itself is a key regulator of a variety of cellular processes, including $\mathrm{G}_{1}$ entry during proliferation $(45,46)$. The high percentage of Th2 cells that fail to incorporate BrdU or express Ki-67 during late chronic infection would be consistent with $\mathrm{G}_{0}$ arrest as a result of altered Rho signaling. The pathways linking repeated stimulation of T cells through TCR with increased expression of GRAIL are unknown at present, but clearly of great interest. Our data from experiments in which the development of hyporesponsiveness was partially prevented by RNAi targeting of GRAIL support the view that GRAIL plays a significant role in this process, but also suggest that other factors may contribute.

Our findings indicate that immunomodulation in schistosomiasis is a reflection of Th2 cell-intrinsic hyporesponsiveness and most readily defined as anergy or adaptive tolerance. We are interested now in determining the long-term significance of Th2 cell hyporesponsiveness, with a particular focus on whether hyporesponsive Th2 cells have the potential to give rise to long-lived memory cells once infection has been resolved. Such a scenario might fit well with the observed development of resistance to reinfection in schistosomiasis patients who have been drug treated (47). Points of additional interest are (a) whether defects in GRAIL upregulation within Th2 cells underlie exacerbated immunopathology associated with poorly modulated immune responses in patients with hepatosplenic schistosomiasis; (b) the relationship between GRAIL expression and the regulation of cytokine production in hypoproliferative Th2 cells; and (c) the possibility that other factors cooperate with GRAIL to limit Th2 cell proliferation. Studies directed toward addressing these issues will generate new insights into the processes that allow the regulation of Th2 responses during chronic schistosomiasis and other chronic helminth infections in which Th2 cell hyporesponsiveness has been documented (20). In addition, these issues are of relevance to the development of therapies for important diseases of the developed world that are mediated by chronic, poorly controlled Th2 responses, such as asthma, allergy, and ulcerative colitis.

\section{Methods}

Animals, infections, and immunizations. BALB/c 4get mice were generated as described previously (32) and maintained at the University of Pennsylvania. We generated 4get Thy 1.1 mice using Thy 1.1 mice originally obtained from L. Turka (University of Pennsylvania). Mice were infected by percutaneous exposure to approximately 20 S. mansoni cercariae (Puerto Rican strain NMRI; Biomedical Research Institute). Schistosome eggs were isolated from livers of infected mice and resuspended at 100,000 eggs/ml in PBS (SigmaAldrich), as described previously (48). Soluble SEA was prepared as described previously (48). Eggs suspended in $25 \mu \mathrm{l}$ PBS were injected into hind footpads, and draining popliteal LNs were analyzed. Procedures were conducted in compliance with federal guidelines, and the University of Pennsylvania Animal Care and Use Committee approved the study protocol.

Cell isolation and culture. Single-cell suspensions $\left(5 \times 10^{6}\right.$ cells $\left./ \mathrm{ml}\right)$ from spleens were cultured in 96 -well plates at $37^{\circ} \mathrm{C}$ and $5 \% \mathrm{CO}_{2}$ in Isocove's DMEM containing $100 \mathrm{U} / \mathrm{ml}$ penicillin, $100 \mu \mathrm{g} / \mathrm{ml}$ streptomycin, $2 \mathrm{mM}$ L-glutamine (all from Mediatech), $5 \times 10^{-5} \mathrm{M} 2-\mathrm{ME}$ (Sigma-Aldrich), and $10 \%$ FCS (HyClone) with or without the addition of $50 \mu \mathrm{g} / \mathrm{ml} \mathrm{SEA}$. Culture supernatants were harvested at $72 \mathrm{~h}$, and cytokine concentrations were measured by ELISAs as described previously (48). In some experiments, splenocytes were labeled with CFSE (Invitrogen), as described previously (49). To analyze hepatic Th2 cells, livers were excised from PBS-perfused animals, chopped, and incubated in RPMI (Mediatech) containing 250 $\mu \mathrm{g} / \mathrm{ml}$ Collagenase D (Roche) and $10 \mu \mathrm{g} / \mathrm{ml}$ DNase I (Sigma-Aldrich) at $37^{\circ} \mathrm{C}$ for $30 \mathrm{~min}$. Liver homogenates were mechanically disrupted through a $100-\mu \mathrm{m}$ cell strainer (BD Biosciences) and centrifuged through $40 \%$ isotonic Percoll/RPMI. The resulting pellet was washed, rbcs were lysed, and remaining cells were used for analyses. For restimulation of sorted Th cells, $5 \times 10^{4} / \mathrm{ml}$ bone marrow-derived DCs, generated as described previously (48), were cultured for $4 \mathrm{~h}$ with SEA $(50 \mu \mathrm{g} / \mathrm{ml})$ prior to the addition of $2.5 \times 10^{5} / \mathrm{ml}$ Th cells. Alternatively, Th cells were added to anti-CD3-coated plates $(0.5 \mu \mathrm{g} /$ well, clone no. $145-2 \mathrm{C} 11$; eBioscience) with or without antiCD28 (5 $\mu \mathrm{g} / \mathrm{ml}$, clone no. 37.51; BD Biosciences). To analyze proliferation, $10 \mathrm{mM} \mathrm{BrdU}$ (BD Biosciences) was added at the start of culture. For 8-d in vitro restimulations, Th cells were mixed with SEA-pulsed DCs as described above, and $3 \mathrm{~d}$ later, additional Ag-pulsed DCs were added to the cultures.

Flow cytometry. Cells were labeled with fluorochrome-conjugated antibodies as described previously (38). Ki-67 expression was detected using a PE Mouse Anti-Human Ki-67 Set (BD Biosciences), which also detects mouse Ki-67. Due to a loss of fluorescence following fixation, EGFP was in some cases detected using rabbit anti-GFP (eBioscience), followed by FITC anti-rabbit IgG antibody (Jackson Immunoresearch Laboratories). Samples were acquired using FACSCalibur, FACSCanto II, or LSR II flow cytometers (BD Biosciences) and analyzed with FlowJo software (version 8.2; Tree Star). All plots shown are on a Logicle scale (50), except for Figure $1 \mathrm{~A}$, which is shown in logarithmic format.

Cell sorting and adoptive transfer. Spleen and mesenteric LN cells were pooled, and $\mathrm{CD}^{+}$cells were presorted using MACS (Miltenyi Biotec) followed by FACS sorting to purify GFP- and $\mathrm{GFP}^{+}$cells using FACSAria or FACSDiva cell sorters (BD Biosciences). Sorted cells were routinely greater than $97 \%$ pure. FACS-sorted $\mathrm{GFP}^{+} \mathrm{CD} 4^{+}$cells $\left(\sim 0.5 \times 10^{6}\right)$ were injected i.v. into congenic animals $1 \mathrm{~d}$ prior to the initiation of BrdU labeling, and spleen cells were analyzed $7 \mathrm{~d}$ later by flow cytometry. Files generated from replicate mice were concatenated within respective groups for display.

In vivo BrdU labeling. Mice were injected i.p. with $1 \mathrm{mg} \mathrm{BrdU} \mathrm{(BD} \mathrm{Biosci-}$ ences or Sigma-Aldrich) at the start of the labeling period and thereafter provided with $0.8 \mathrm{mg} / \mathrm{ml} \mathrm{BrdU}$ in their drinking water. Fresh water with BrdU was provided every $2-3$ days.

Real-time RT-PCR. RNA was isolated using RNAeasy (Qiagen), treated with TURBO DNA-Free (Ambion), and used to synthesize cDNAs using Oligo(dT) (Promega) and Superscript II polymerase (Invitrogen). Real-time RT-PCR analysis was performed using SYBR-green (Applied Biosystems) on an ABI 7500 Fast Real-time PCR system (Applied Biosystems). Relative expression was calculated using the $2 \Delta \Delta \mathrm{Ct}$ method normalized to hypoxanthine-guanine phosphoribosyltransferase (HPRT). Dissociation curves were generated to verify the presence of a single amplicon. Qiagen Realtime primers were used for GRAIL. HPRT primers were 5'-CTCCGCCGGCTTCCTCCTCA-3' and 5'-ACCTGGTTCATCATCGCTAATC-3'.

GRAIL knockdown. A PCR-based strategy was used to generate a shRNA to target GRAIL, as described previously (51). A microRNA-30-adapted shRNAmir retroviral vector, MSCV-LTRmiR30-PIG (LMP; Open Biosciences), was modified to express hCD8 (gift of R. Jones, McGill University, Montreal, Quebec, Canada) (51). A 97-mer template oligo containing the mir30 and shRNA sequences was amplified using PCR and subcloned into the LMP-hCD8 vector. Retrovirus was produced in $293 \mathrm{~T}$ cells by cotransfection of the construct with helper plasmid containing gag, pol, and env genes using Lipofectamine 2000 (Invitrogen). Supernatants containing retrovirus were collected at $48 \mathrm{~h}$, supplemented with $4 \mu \mathrm{g} / \mathrm{ml}$ polybrene, and used for spin infections $(500 \mathrm{~g}$ for $1 \mathrm{~h}$ ) of freshly purified $\mathrm{GFP}^{+} \mathrm{CD} 4^{+}$cells in 12 -well plates. After infection, cells were replated with SEA-pulsed DCs for $8 \mathrm{~d}$ as described above. Following stimulation, $\mathrm{GFP}^{+} \mathrm{CD} 4^{+} \mathrm{hCD}^{+}$cells were FACS purified. For in vivo experiments, $\mathrm{CD}^{+} \mathrm{GFP}^{+}$cells were FACS purified from 4get Thy1.1 and 4get Thy1.2 mice that had been injected with eggs $5 \mathrm{~d}$ earlier, and spin infected with 
retrovirus. The 4get Thy1.1 cells received the GRAILhp retrovirus, while 4get Thy 1.2 cells received LUChp. After spin infection, cells were placed into culture with SEA-pulsed DCs for $1 \mathrm{~d}$ (initiating cell division and thus essential for optimal transduction), harvested, mixed, and transferred into Balb/c mice infected for over $10 \mathrm{wk}$. Recipient mice were labeled with BrdU for $3 \mathrm{~d}$ immediately prior to analysis at day 11 . The effectiveness of the shRNA was assessed in Th2 cells from mice injected $8 \mathrm{~d}$ previously with eggs. The target sequence of GRAIL was obtained from RNAi Codex (http://codex.cshl.edu), namely, the HP_127117 oligo sequence TGCTGTTGACAGTGAGCGCGGAGTAGCTGTAATCACTTTATAGTGAAGCCACAGATGTATAAAGTGATTACAGCTACTCCATGCCTACTGCCTCGGA. The firefly luciferase hairpin oligo sequence used was CTCGAGAAGGTATATTGCTGTTGACAGTGAGCGAGCTCCCGTGAATTGGAATCCTAGTGAAGCCACAGATGTAGGATTCCAATTCAGCGGGAGCCTGCCTACTGCCTCGGAATTC.

Statistics. To calculate the significance of differences between means, 2-tailed Student's $t$ test was used. All experiments were repeated 2 or more times with similar results. A $P$ value less than 0.05 was considered significant.

\section{Acknowledgments}

The authors are grateful to Andrew Wells, Hao Shen, Phillip Scott, John Wherry, Erika Pearce, and Rusty Jones for helpful discussions; to Elia Tait for critical reading of the manuscript; to Euihye Jung and Tori Freitas for assistance with the parasites; and to the Flow Cytometry and Cell Sorting Core Facility for expert technical assistance. This work was supported by NIH grants AI32573 to E.J. Pearce and AI072296 to M. Mohrs and by a Humans Frontier award to C.M. Krawczyk. Schistosome life stages were provided by NIAID contract NO155270.

Received for publication June 18, 2008, and accepted in revised form January 7, 2009.

Address correspondence to: Edward J. Pearce, Department of Pathobiology, Room 318 Hill Pavilion, 380 South University Avenue, Philadelphia, Pennsylvania 19104-4539, USA. Phone: (215) 5733493; Fax: (215) 746-2295; E-mail: ejpearce@mail.med.upenn.edu.
1. Schwartz, R.H. 2003. T cell anergy. Annu. Rev. Immunol. 21:305-334.

2. Shin, H., and Wherry, E.J. 2007. CD8 T cell dysfunction during chronic viral infection. Curr. Opin. Immunol. 19:408-415.

3. Mathers, C.D., Ezzati, M., and Lopez, A.D. 2007. Measuring the burden of neglected tropical diseases: the global burden of disease framework. PLoS Negl. Trop. Dis. 1:e114.

4. Hotez, P.J., et al. 2008. Helminth infections: the great neglected tropical diseases. J. Clin. Invest. 118:1311-1321.

5. van der Werf, M.J., et al. 2003. Quantification of clinical morbidity associated with schistosome infection in sub-Saharan Africa. Acta Trop. 86:125-139.

6. Hotez, P.J., Ottesen, E., Fenwick, A., and Molyneux, D.H. 2006. The neglected tropical diseases: the ancient afflictions of stigma and poverty and the prospects for their integrated control and elimination. In Hot topics in infection and immunity in children III. Kluwer Academic/Plenum Publishers. New York, New York, USA. 23-33.

7. Grzych, J.M., et al. 1991. Egg deposition is the major stimulus for the production of Th2 cytokines in murine schistosomiasis mansoni. J. Immunol. 146:1322-1327.

8. Pearce, E.J., Caspar, P., Grzych, J.M., Lewis, F.A., and Sher, A. 1991. Downregulation of Th1 cytokine production accompanies induction of Th2 responses by a parasitic helminth, Schistosoma mansoni. J. Exp. Med. 173:159-166.

9. Buchanan, R.D., Fine, D.P., and Colley, D.G. 1973. Schistosoma mansoni infection in mice depleted of thymus-dependent lymphocytes. II. Pathology and altered pathogenesis. Am. J. Pathol. 71:207-218.

10. Lucas, S., et al. 1980. The pathological effects of immunosuppression of Schistosoma mansoniinfected mice, with particular reference to survival and hepatotoxicity after thymectomy and treatment with antithymocyte serum, and treatment with hydrocortisone acetate. Trans. $R$ Soc. Trop. Med. Hyg. 74:633-643.

11. Brunet, L.R., Finkelman, F.D., Cheever, A.W., Kopf, M.A., and Pearce, E.J. 1997. IL-4 protects against TNF-alpha-mediated cachexia and death during acute schistosomiasis. J. Immunol. 159:777-785.

12. Fallon, P.G., Richardson, E.J., McKenzie, G.J., and McKenzie, A.N. 2000. Schistosome infection of transgenic mice defines distinct and contrasting pathogenic roles for IL-4 and IL-13: IL-13 is a profibrotic agent. J. Immunol. 164:2585-2591.

13. Herbert, D.R., et al. 2004. Alternative macrophage activation is essential for survival during schistosomiasis and downmodulates $T$ helper 1 responses and immunopathology. Immunity. 20:623-635.

14. Fitzsimmons, C.M., et al. 2005. Molecular characterization of omega-1: a hepatotoxic ribonuclease from Schistosoma mansoni eggs. Mol. Biochem. Parasitol. 144:123-127.

15. Chiaramonte, M.G., Donaldson, D.D., Cheever, A.W., and Wynn, T.A. 1999. An IL-13 inhibitor blocks the development of hepatic fibrosis during a T-helper type 2-dominated inflammatory response. J. Clin. Invest. 104:777-785.

16. Wynn, T.A., Thompson, R.W., Cheever, A.W., and Mentink-Kane, M.M. 2004. Immunopathogenesis of schistosomiasis. Immunol. Rev. 201:156-167.

17. Domingo, E.O., and Warren, K.S. 1968. Endogenous desensitization: changing host granulomatou response to schistosome eggs at different stages of infection with schistosoma mansoni. Am. J. Pathol. 52:369-379.

18. Boros, D.L., Pelley, R.P., and Warren, K.S. 1975. Spontaneous modulation of granulomatous hypersensitivity in schistosomiasis mansoni. J. Immunol. 114:1437-1441.

19. Phillips, S.M., and Colley, D.G. 1978. Immunologic aspects of host responses to schistosomiasis: resistance, immunopathology, and eosinophil involvement. Prog. Allergy. 24:49-182.

20. Maizels, R.M., et al. 2004. Helminth parasites--masters of regulation. Immunol. Rev. 201:89-116.

21. Colley, D.G., et al. 1986. Immune responses during human schistosomiasis. XII. Differential responsiveness in patients with hepatosplenic disease. Am. J. Trop. Med. Hyg. 35:793-802.

22. Wilson, M.S., et al. 2007. Immunopathology of schistosomiasis. Immunol. Cell Biol. 85:148-154.

23. Rumbley, C.A., Sugaya, H., Zekavat, S.A., Perrin, P.J., and Phillips, S.M. 2001. Elimination of lymphocytes, but not eosinophils, by Fas-mediated apoptosis in murine schistosomiasis. Am. J. Trop. Med. Hyg. 65:442-449.

24. Lundy, S.K., Lerman, S.P., and Boros, D.L. 2001. Soluble egg antigen-stimulated T helper lymphocyte apoptosis and evidence for cell death mediated by FasL $(+) \mathrm{T}$ and $\mathrm{B}$ cells during murine Schistosoma mansoni infection. Infect. Immun. 69:271-280.

25. Rutitzky, L.I., Mirkin, G.A., and Stadecker, M.J. 2003. Apoptosis by neglect of CD4+ Th cells in granulomas: a novel effector mechanism involved in the control of egg-induced immunopathology in murine schistosomiasis. J. Immunol. 171:1859-1867.

26. Stadecker, M.J., Kamisato, J.K., and Chikunguwo, S.M. 1990. Induction of T helper cell unresponsiveness to antigen by macrophages from schistosomal egg granulomas. A basis for immunomodulation in schistosomiasis? J. Immunol. 145:2697-2700.
27. Smith, P., et al. 2004. Schistosoma mansoni worms induce anergy of $\mathrm{T}$ cells via selective up-regulation of programmed death ligand 1 on macrophages. J. Immunol. 173:1240-1248.

28. Flores Villanueva, P.O., Chikunguwo, S.M., Harris, T.S., and Stadecker, M.J. 1993. Role of IL-10 on antigen-presenting cell function for schistosomal egg-specific monoclonal $\mathrm{T}$ helper cell responses in vitro and in vivo. J. Immunol. 151:3192-3198.

29. King, C.L., et al. 1996. Cytokine control of parasitespecific anergy in human urinary schistosomiasis. IL-10 modulates lymphocyte reactivity. J. Immunol. 156:4715-4721.

30. Sadler, C.H., Rutitzky, L.I., Stadecker, M.J., and Wilson, R.A. 2003. IL-10 is crucial for the transition from acute to chronic disease state during infection of mice with Schistosoma mansoni. Eur. J. Immunol. 33:880-888.

31. van Den Biggelaar, A.H., et al. 2000. Chronic schistosomiasis: dendritic cells generated from patients can overcome antigen-specific $\mathrm{T}$ cell hyporesponsiveness. J. Infect. Dis. 182:260-265.

32. Mohrs, M., Shinkai, K., Mohrs, K., and Locksley, R.M. 2001. Analysis of type 2 immunity in vivo with a bicistronic IL-4 reporter. Immunity. 15:303-311.

33. Mohrs, K., Harris, D.P., Lund, F.E., and Mohrs, M. 2005. Systemic dissemination and persistence of Th2 and type 2 cells in response to infection with a strictly enteric nematode parasite. J. Immunol. 175:5306-5313.

34. Anandasabapathy, N., et al. 2003. GRAIL: an E3 ubiquitin ligase that inhibits cytokine gene transcription is expressed in anergic CD4+ T cells. Immunity. 18:535-547.

35. Seroogy, C.M., et al. 2004. The gene related to anergy in lymphocytes, an E3 ubiquitin ligase, is necessary for anergy induction in CD4 T cells. J. Immunol. 173:79-85.

36. Gerdes, J., et al. 1984. Cell cycle analysis of a cell proliferation-associated human nuclear antigen defined by the monoclonal antibody Ki-67.J. Immunol. 133:1710-1715.

37. Vella, A.T., and Pearce, E.J. 1992. CD4+ Th2 response induced by Schistosoma mansoni eggs develops rapidly, through an early, transient, Th0-like stage. J. Immunol. 148:2283-2290.

38. Taylor,J.J., Mohrs, M., and Pearce, E.J. 2006. Regulatory $\mathrm{T}$ cell responses develop in parallel to $\mathrm{Th}$ responses and control the magnitude and phenotype of the Th effector population. J. Immunol. 176:5839-5847.

39. Mobley, J.L., and Dailey, M.O. 1992. Regulation of adhesion molecule expression by CD8 T cells in vivo. I. Differential regulation of gP90MEL-14 (LECAM-1), Pgp-1, LFA-1, and VLA-4 alpha during the differ- 
entiation of cytotoxic T lymphocytes induced by allografts. J. Immunol. 148:2348-2356.

40. Ziegler, S.F., Ramsdell, F., and Alderson, M.R. 1994. The activation antigen CD69. Stem Cells. 12:456-465.

41. Pearce, E.J., and MacDonald, A.S. 2002. The immunobiology of schistosomiasis. Nat. Rev. Immunol. 2:499-511.

42. Wherry, E.J., et al. 2007. Molecular signature of CD8+ T cell exhaustion during chronic viral infection. Immunity. 27:670-684.

43. MacKenzie, D.A., et al. 2007. GRAIL is up-regulated in CD4+CD25+ T regulatory cells and is sufficient for conversion of $\mathrm{T}$ cells to a regulatory phenotype. J. Biol. Chem. 282:9696-9702.

44. Su, L., Lineberry, N., Huh, Y., Soares, L., and Fath- man, C.G. 2006. A novel E3 ubiquitin ligase substrate screen identifies Rho guanine dissociation inhibitor as a substrate of gene related to anergy in lymphocytes. J. Immunol. 177:7559-7566.

45. Olson, M.F., Ashworth, A., and Hall, A. 1995. An essential role for Rho, Rac, and Cdc42 GTPases in cell cycle progression through G1. Science. 269:1270-1272.

46. Etienne-Manneville, S., and Hall, A. 2002. Rho GTPases in cell biology. Nature. 420:629-635.

47. McManus, D.P., and Loukas, A. 2008. Current status of vaccines for schistosomiasis. Clin. Microbiol. Rev. 21:225-242.

48. MacDonald, A.S., Straw, A.D., Bauman, B., and Pearce, E.J. 2001. CD8- dendritic cell activation status plays an integral role in influencing Th2 response development. J. Immunol. 167:1982-1988. 49. Wells, A.D., Gudmundsdottir, H., and Turka, L.A. 1997. Following the fate of individual T cells throughout activation and clonal expansion. Signals from $\mathrm{T}$ cell receptor and CD28 differentially regulate the induction and duration of a proliferative response. J. Clin. Invest. 100:3173-3183.

50. Herzenberg, L.A., Tung, J., Moore, W.A., Herzenberg, L.A., and Parks, D.R. 2006. Interpreting flow cytometry data: a guide for the perplexed. Nat. Immunol. 7:681-685.

51. Paddison, P.J., et al. 2004. Cloning of short hairpin RNAs for gene knockdown in mammalian cells. Nat. Methods. 1:163-167. 